\title{
Caracterización microestructural de mármol de una cantera localizada en el Municipio de Tepexi de Rodríguez, Estado de Puebla
}

\author{
Microstructural characterization of marble from a quarry located in the Municipality of Tepexi de Rodríguez, State of \\ Puebla
}

\author{
E. Rubio-Rosas (DD a , L. S. Villaseñor-Cerón (iD b , E. Salinas-Rodríguez (iD b , J. Hernández-Ávila (D b \\ O.A. Acevedo-Sandoval iD c , V. Rodríguez-Lugo iD b

\begin{abstract}
a Benemérita Universidad Autónoma de Puebla, Prolongación de la 24 Sur y San Claudio, Ciudad Universitaria, Sn Manuel, 72570 Puebla, México.
${ }^{b}$ Área Académica de Ciencias de la Tierra y Materiales, Instituto de Ciencias Básicas e Ingeniería, Universidad Autónoma del Estado de Hidalgo, Carretera PachucaTulancingo Km. 4.5, 42184, México.

${ }^{c}$ Área Académica de Química, Universidad Autónoma del Estado de Hidalgo, Unidad Universitaria, km 4.5 Carretera Pachuca-Tulancingo, C.P. 42184 Pachuca-
\end{abstract} \\ Hidalgo, México
}

\begin{abstract}
Resumen
En el presente trabajo se llevó a cabo la caracterización microestructural de mármol (rocas metamórficas no foliadas) procedente de una cantera localizada en el Municipio de Tepexi Rodriguez en el Estado de Puebla. Los resultados por difracción de rayos X y por espectroscopia en el infrarrojo (FTIR), permitieron confirmar la presencia mayoritaria de la fase calcita, sin embargo, por la presencia de elementos químico minoritarios como $\mathrm{Si}, \mathrm{Al}$ y $\mathrm{Mg}$, detectados por espectroscopia de dispersión de energía de rayos X, es posible asegurar la que existen otras fases cristalinas en concentraciones inferiores al $1 \%$, que no fueron detectadas a consecuencia de límite de detección de los equipos utilizados. Por Microscopia electrónica de barrido, fue posible observar cambios significativos en el tamaño de partícula en cada una de las muestras obtenidas de cada estrato estudiado, lo que permite predecir cambios en la resistencia a los esfuerzos mecánicos.
\end{abstract}

Palabras Clave:

Mármol, calcita, carbonato de calcio.

\begin{abstract}
In the present work, the microstructural characterization of non-foliated metamorphic rocks (marble) from a quarry located in the Municipality of Tepexi Rodriguez in the State of Puebla was carried out. The results by X-ray diffraction and by infrared spectroscopy (FTIR), allowed to confirm the majority presence of the calcite phase, however, due to the presence of minor chemical elements such as $\mathrm{Si}, \mathrm{Al}$ and $\mathrm{Mg}$, detected by energy-dispersive X-ray spectroscopy, it is possible to ensure the presence of other crystalline phases in concentrations lower than $1 \%$, which were not detected as a result of the detection limit of the equipment used. By scanning electron microscopy, it was possible to observe significant changes in particle size in each of the samples obtained from each stratum studied, which allows predicting changes in resistance to mechanical stress.
\end{abstract}

Keywords:

Marble, calcite, calcium carbonate.

\section{Introducción}

México es uno de los países más importantes a escala mundial en cuanto a riqueza minera se refiere, tanto por cantidad y calidad de especies minerales como por su abundancia minera y por la diversidad de tipologías de depósitos minerales encontradas a lo largo de su territorio, destacando la extracción de plata, cobre y oro. Sin embargo, también tiene un gran potencial para la explotación de los minerales no metálicos. Es el caso del mármol en el Estado de Puebla. particularmente en el municipio de Tepexi de Rodríguez, que se encuentra inserto en la Mixteca poblana y que comenzó a experimentar un crecimiento económico importante a partir de la década de los setenta del siglo XX, cuando la industria de la extracción y transformación de mármol llegó a esa región para darle una nueva dinámica a la economía local.

\footnotetext{
*Autor para la correspondencia: ventura.r165@gmail.com herjuan@uaeh.edu.mx (Juan Hernández Ávila) y ventura.rl65@gmail.com (Ventura Rodríguez Lugo)

Correo electrónico: efrainrubio@yahoo.com (Efraín Rubio Rosas), leslysabina@gmail.com (Lesly Sabina Villaseñor Cerón), salinasr@uaeh.edu.mx (Eleazar Salinas Rodríguez), 
Rocas metamórficas no foliadas: Son rocas en donde los granos minerales no muestran una orientación preferencial distinguible, en lugar de esto, presentan un mosaico de minerales un tanto equidimensionales que son el resultado del metamorfismo de contacto o regional en rocas donde no hay presencia de minerales laminados o alargados. Un ejemplo de estas rocas y de gran importancia comercial para Puebla, es el mármol, que es generada por el metamorfismo de la caliza y dolomía. La calcita o bien llamado carbonato de calcio $\left(\mathrm{CaCO}_{3}\right)$ es el constituyente principal de las calizas (Morales Tassinai et al. 2017b; Morales Tassinai, Velasco Ordónez, and Ramírez Peña 2020). La calcita es formada por la precipitación del carbonato cálcico, como se muestra en la ecuación (1):

$$
\mathrm{Ca}^{2+}+2\left(\mathrm{HCO}_{3}^{-}\right) \rightarrow \mathrm{CaCO}_{3}+\mathrm{CO}_{2}+\mathrm{H}_{2} \mathrm{O}
$$

Esta reacción expresa claramente cómo al disminuir el contenido de $\mathrm{CO}_{2}$ de las aguas se produce dicha precipitación. La disminución de $\mathrm{CO}_{2}$ en el agua puede ser consecuencia de un calentamiento de ésta, de una disminución de presión, de la actividad fotosintética de las algas, de la evaporación, del cese de respiración de organismos vivos, o de una combinación de dos o más de estas causas (Rioja, Domingo, and Calzada 2016).

La calcita tiene una cristalografía romboédrica, misma que presenta una forma polimórfica; la cual es el aragonito con una cristalografía ortorrómbica, y este mineral es menos estable que la calcita y con menos impurezas. Se forma en condiciones fisicoquímicas muy determinadas de baja temperatura, en depósitos superficiales (Morales Tassinai, Velasco Ordónez, and Ramírez Peña 2019). A presión ordinaria, el aragonito empieza a convertirse en calcita, por inversión a los $400^{\circ} \mathrm{C}$; en presencia del agua o de soluciones que contengan $\mathrm{CaCO}_{3}$ disuelto, la inversión puede tener lugar a la temperatura ambiente. Una tercera forma del $\mathrm{CaCO}_{3}$ algunas veces llamada vaterita, se conoce, pero no es de importancia geológica (Hernández et al. 2014).

El mármol es uno de los minerales que tiene un interés práctico en la construcción, tanto en bruto como pulimentado (Corinaldesi, Moriconi, and Naik 2010). Los mármoles representan también la materia prima más frecuente para su empleo en la escultura, por lo consiguiente las características y propiedades del material influyen significativamente en el campo de aplicación del mismo (Morales Tassinai et al. 2017a).

Es por lo que el presente trabajo se enfoca en la caracterización del mármol extraído de Tepexi de Rodríguez de Estado de Puebla, México, a través de microscopia electrónica de barrido, espectroscopia de dispersión de energía de rayos X, difracción de rayos X y espectroscopia en el infrarrojo. Debido a que es uno de los lugares donde la extracción de mármol representa la principal actividad económica (Miranda-Trejo et al. 2015; Tlatempa et al. 2011), el conocimiento de las características microestructurales permitirá generar un mayor valor agregado a la explotación de este tipo de materiales, lo que contribuirá a determinar su calidad y permitiendo mayores aplicaciones, y en consecuencia un mayor beneficio económico.

\section{Metodología}

Cuatro muestras de mármol de aproximadamente un kilogramo de peso fueron extraídas de una cantera en el municipio de Tepexi de Rodríguez, Puebla perteneciente a la región Mixteca.

En la figura 1 se presenta un mapa del municipio y las coordenadas geográficas de la cantera de mármol $\left(18^{\circ} 35^{\prime} 48^{\prime \prime} \mathrm{N}\right.$, $97^{\circ} 54^{\prime} 54^{\prime}$ O). En la figura 2, se presenta una vista general del afloramiento de mármol que ha sido explotado comercialmente. En la figura 3, se presentan las etiquetas M1, M2, M3 y M4 que corresponden a cuatro estratos de los cuales se retiraron cuatro muestras respectivamente para su caracterización.

La muestra M1 se recolecto en la base del afloramiento con el apoyo de un piolet ( 0 metros), las muestras M2, M3 y M4 se recolectaron de manera ascendente vertical hacia el bloque techo (4, 6.88 y 9.03 metros respectivamente), tal y como se ilustra en la figura 3 .

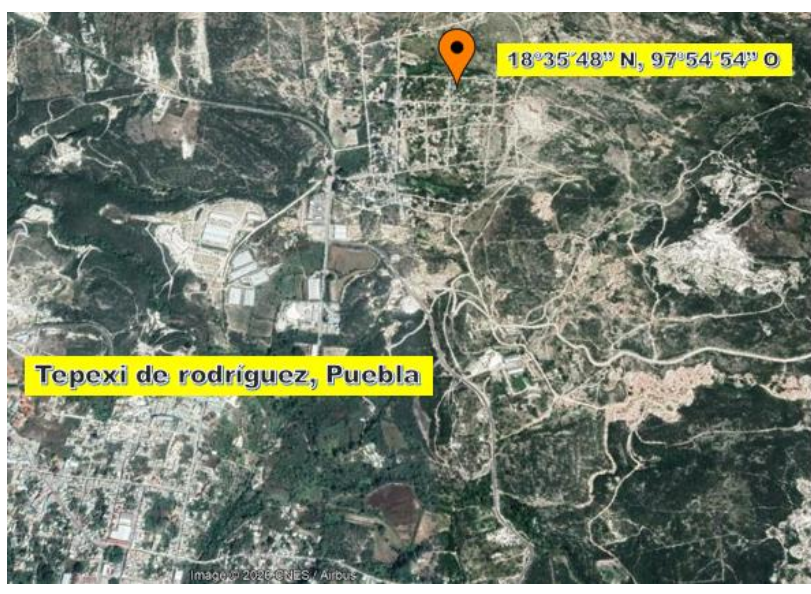

Figura 1. Geolocalización de la cantera de mármol en el Municipio de Tepexi de Rodríguez, Puebla.

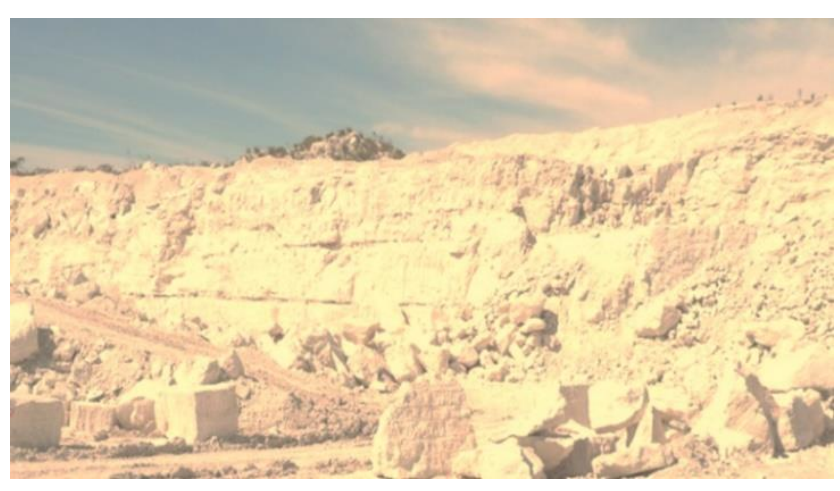

Figura 2. Vista general del afloramiento de mármol. 


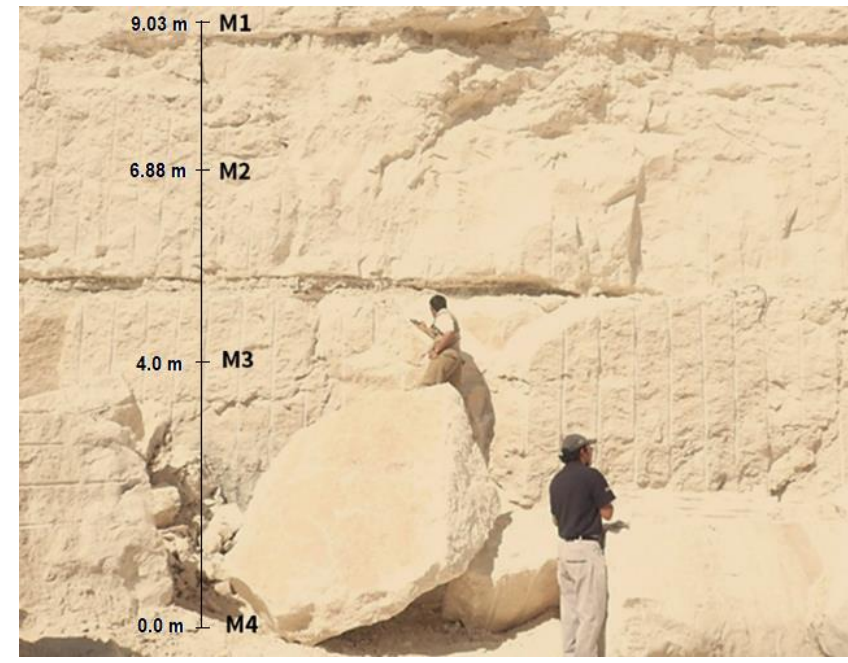

Figura 3. Vista del afloramiento de mármol, donde se presentan los puntos donde fueron recolectadas las muestras M1, M2, M3 y M4 respectivamente.

Las muestras se caracterizaron por Microscopia Electrónica de Barrido (MEB), utilizando un microscopio de la marca JEOL modelo JSM-6610LV. Las imágenes se formaron utilizando electrones retrodispersados, magnificación de 2500X, un potencial de aceleración de $20 \mathrm{kV}$ y $30 \mathrm{~Pa}$ de presión. Las muestras fueron directamente analizadas sin previa preparación.

La distribución de tamaño de partícula y tamaño de partícula promedio se realizó utilizando las imágenes MEB de diferentes zonas de cada una de las muestras, midiendo más de 150 partículas por muestra y procesadas por el software ImageJ.

Se realizó un análisis químico elemental por dispersión de energía de rayos $\mathrm{X}$ (EDS) a cada una de las muestras, utilizando una sonda marca Oxford modelo INCA energy $250+$, con resolución de $137 \mathrm{eV}$ y detector de $10 \mathrm{~mm}^{2}$, las condiciones de la operación fueron las siguientes: 1 min de radiación por medición, 3 mediciones promediadas por muestra, $10 \mathrm{~mm}$ distancia de trabajo, un spot size de 60. El MEB operó a alto vacío y $20 \mathrm{KV}$.

Para identificar las fases cristalinas se utilizó la técnica de difracción de rayos $\mathrm{X}$ (DRX) en polvos, utilizando un difractómetro marca Bruker modelo D8 Discover. Las muestras se pulverizaron en un mortero de ágata y se colocaron en una portamuestra, comprimiendo el polvo hasta quedar perfectamente compacto. Los difractogramas se tomaron en el rango $2 \theta$ de $10^{\circ}$ a $80^{\circ}$, empleando un tiempo de $1.0 \mathrm{seg}$ en cada paso y un tamaño de paso de $0.02^{\circ}$, usando radiación CuK $\alpha$ de $\lambda=1.5406 \AA$, operado a $35 \mathrm{kV}$ y $25 \mathrm{~mA}$, las fases cristalinas se identificaron empleando la base de datos PDF4+ del 2013 del Centro Internacional de Datos de Difracción (ICDD).

Finalmente, las muestras se caracterizaron por Espectroscopia Infrarroja por Transformadas de Fourier (FTIR), utilizando un espectrómetro de Infrarrojo, marca Bruker modelo Vertex70. Para el análisis, las muestras se pulverizaron en un mortero ágata y se depositaron para su análisis en la superficie de diamante del sistema de Reflectancia Total Atenuada (ATR), el análisis fue realizado en el intervalo espectral entre $4000-400 \mathrm{~cm}^{-1}$. El instrumento cuenta con una resolución espectral fija de $4 \mathrm{~cm}^{-1}$.

\section{Resultados y Discusión}

Los colores del mármol más representativos en todo el mundo son el: mármol blanco, crema, negro, rojo, amarillo, marrón y verde. Evidentemente existen innumerables tipos de variedades en tonos de mármol, pero éstos son los más utilizados en el mundo (Semar, 2020). En este sentido, podemos apreciar en la Figura 4, las cuatro muestras que corresponde a cada uno de los estratos, en donde se puede apreciar que las cuatro presentan un tono crema, con ligeros cambio en intensidad, particularmente en la muestra 3, que es ligeramente más café que las otras tres, presenta vetas lineales que cubren parcialmente la superficie y son grises. A diferencia de las otras tres, en la muestra 4 , se puede apreciar que las vetas son muy cortas y curvas.
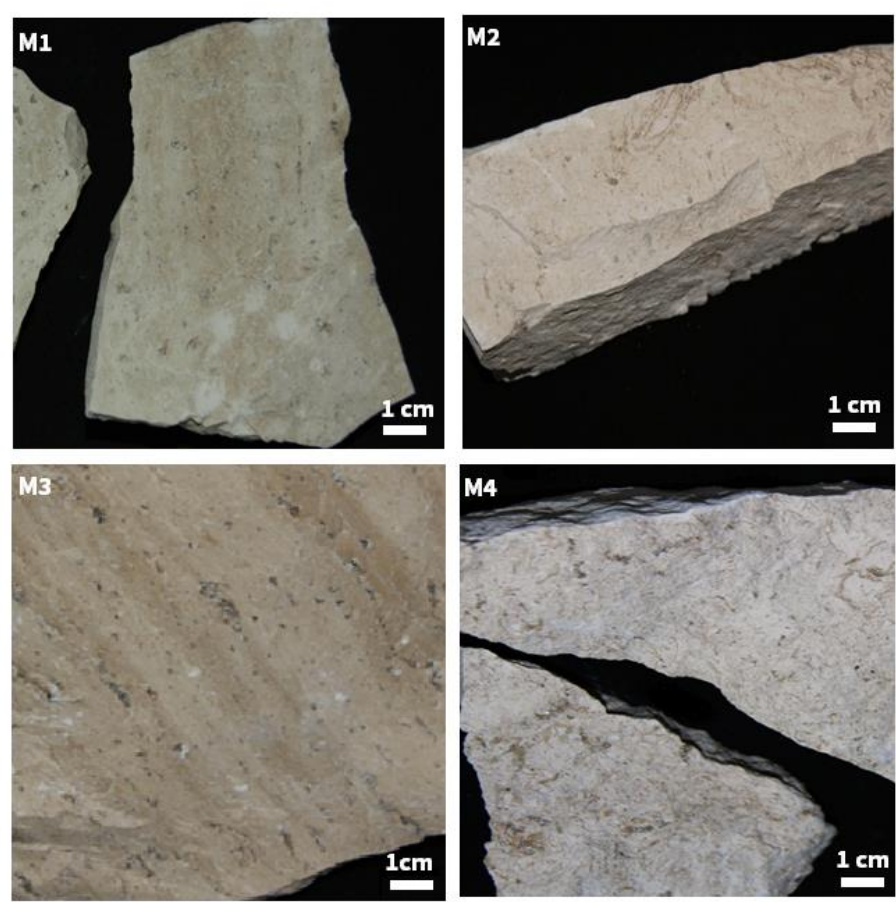

Figura 4. Muestras representativas de mármol, de los cuatro estratos recuperados de una cantera de Tepexi de Rodriguez, Puebla.

En las figuras 5, 6, 7 y 8, se presentan los análisis por MEB en donde es posible apreciar la morfología microestructural de cada muestra, acompañado de su análisis químico elemental correspondiente obtenido por dispersión de energía de rayos $\mathrm{X}$. En la figura 5 a), se presenta una microfotografía de la muestra M1, donde se aprecia que el mármol presenta una morfología granular con tamaños de granos entre $1 \mu \mathrm{m}$ y $14 \mu \mathrm{m}$, con un tamaño promedio de $2.35 \mu \mathrm{m}$, tal y como se puede observar en la figura $5 \mathrm{~b}$ ), es claro que la gran mayoría de las partículas que se observan tienen un tamaño inferior a las 8 micras, sin embargo, existen partículas de mayor tamaño entre 8 y 14 micras; en general los granos están estrechamente unidos, y se observa una reducida presencia de poros.

La composición química elemental de la muestra, se presenta en la figura $5 \mathrm{c}$ ) como una gráfica de pastel, se aprecia la presencia mayoritaria de $\mathrm{C}, \mathrm{Ca}$ y $\mathrm{O}$ que forman el carbonato de calcio, y la presencia minoritaria de $\mathrm{Si}, \mathrm{Al}$ y $\mathrm{Mg}$ con $1.29 \%, 0.5$ $\%$ y $0.16 \%$ en peso respectivamente. 


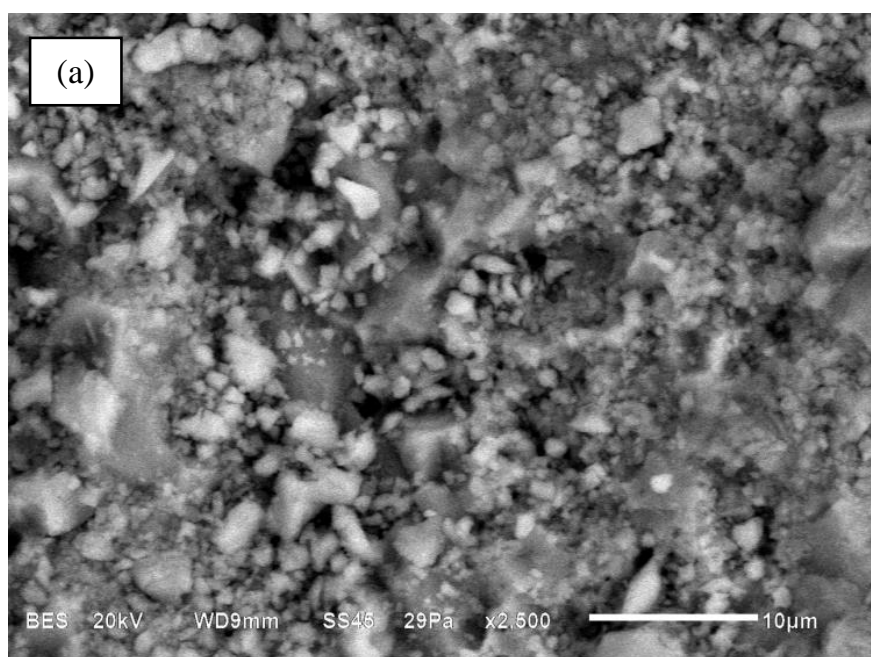

(b)

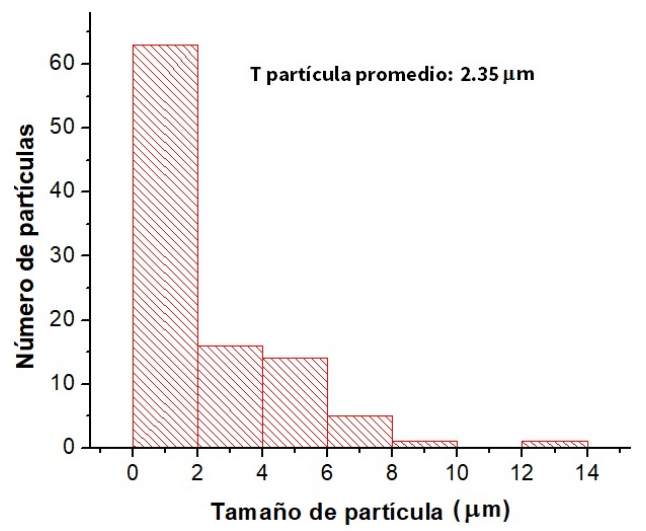

(c)

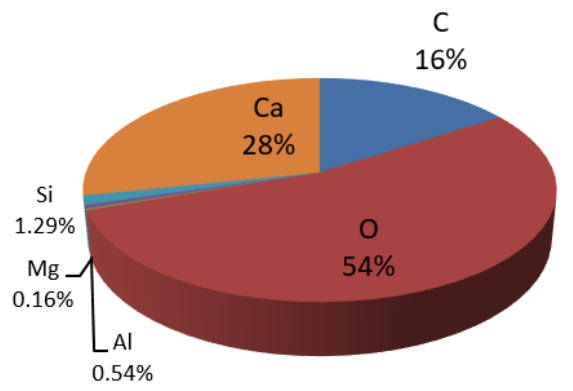

Figura 5. (a) Micrografía de la muestra M1, (b) Histograma del tamaño de grano correspondiente a la muestra M1, (c) Análisis químico elemental en \% en peso de la muestra M1.

En la figura 6 a), se presenta una microfotografía de la muestra M2, donde se aprecia que el mármol presenta una morfología granular con tamaños de granos entre $0.5 \mu \mathrm{m}$ y $5 \mu \mathrm{m}$, con un tamaño promedio de $2.2 \mu \mathrm{m}$, tal y como se puede observar en la figura $6 \mathrm{~b}$ ), a diferencia de la muestra M1, no se detectaron partículas mayores a 5 micras, mostrando una aglomeración de las partículas con una reducida presencia de poros.

La composición química elemental de la muestra, se presenta en la figura 6 c) como una gráfica de pastel, se aprecia la presencia mayoritaria de $\mathrm{C}, \mathrm{Ca}$ y $\mathrm{O}$ que forman el carbonato de calcio, y la presencia minoritaria de $\mathrm{Si}$ y $\mathrm{Al}$ con $1.26 \%$ y $0.56 \%$ en peso respectivamente, no se detectó la presencia de $\mathrm{Mg}$ (probablemente la presencia de este elemento se encuentre por debajo del límite de detección del equipo.
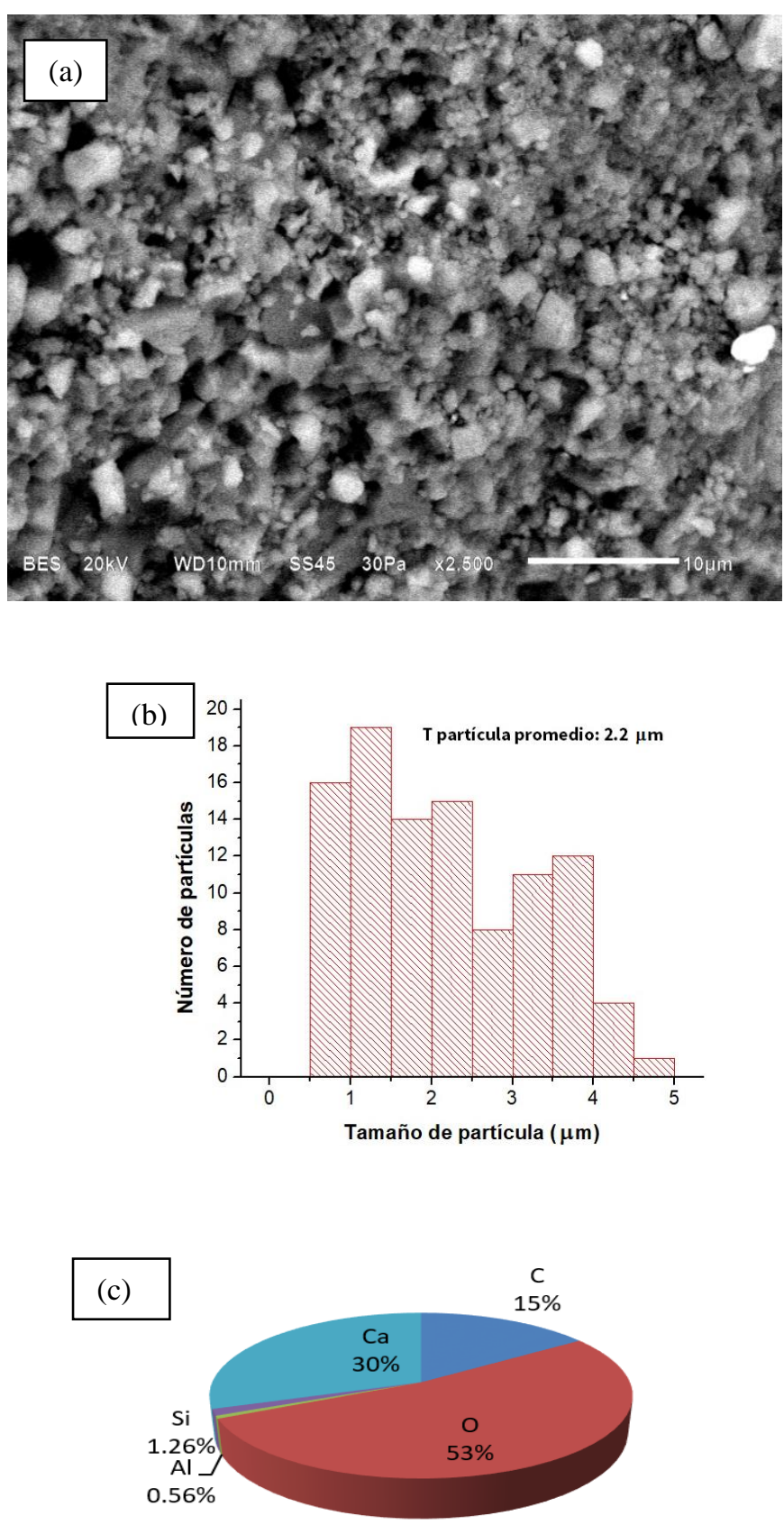

Figura 6. (a) Micrografía de la muestra 2, (b) Histograma del tamaño de grano correspondiente a la muestra M2, (c) Análisis químico elemental en \% en peso de la muestra M2.

En la figura 7 a), se presenta una microfotografía de la muestra M3, donde se aprecia que el mármol presenta una morfología granular con tamaños de granos entre $0.5 \mu \mathrm{m}$ y $5 \mu \mathrm{m}$, con un tamaño promedio de $1.3 \mu \mathrm{m}$, tal y como se puede observar en la figura $7 \mathrm{~b}$ ), es la muestra que presenta el menor tamaño de partícula, no se detectaron partículas mayores a 5 micras, mostrando una aglomeración de las partículas con una reducida presencia de poros.

La composición química elemental de la muestra, se presenta en la figura 7 c) como una gráfica de pastel, se aprecia la presencia mayoritaria de $\mathrm{C}, \mathrm{Ca}$ y $\mathrm{O}$ que forman el carbonato de calcio, y la presencia minoritaria de $\mathrm{Si} 0.56 \%$ en peso 
respectivamente, no se detectó la presencia de otro elemento, aunque es posible que se encuentren por debajo del límite de detección del equipo.
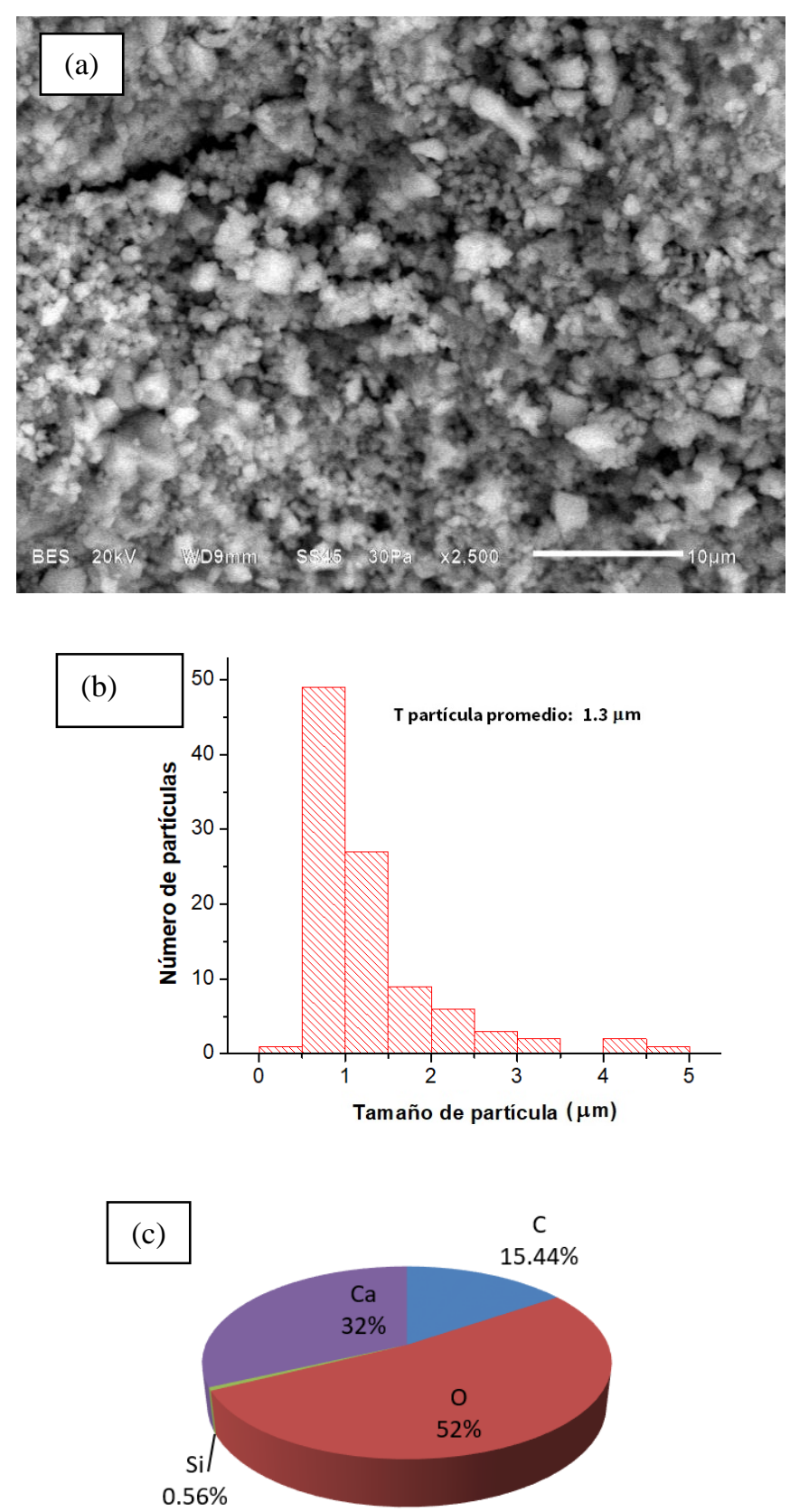

Figura 7. (a) Micrografía de la muestra M3, (b) Histograma del tamaño de grano correspondiente a la muestra M3, (c) Análisis químico elemental en $\%$ en peso de la muestra M3.

En la figura 8 a), se presenta una microfotografía de la muestra M4, donde se aprecia que el mármol presenta una morfología granular con tamaños de grano entre $0.5 \mu \mathrm{m}$ y $30 \mu \mathrm{m}$, con un tamaño promedio de $5.2 \mu \mathrm{m}$, tal y como se puede observar en la figura $8 \mathrm{~b}$ ), es la muestra con un tamaño mayor de grano en que las partículas mayores a 25 micras ocupan la mayor parte del volumen, las partículas se encuentran aglomeradas con una reducida presencia de poros.
La composición química elemental de la muestra, se presenta en la figura 8 c) como una gráfica de pastel, se aprecia la presencia mayoritaria de $\mathrm{C}, \mathrm{Ca}$ y $\mathrm{O}$ que forman el carbonato de calcio, y la presencia minoritaria de $\mathrm{Si}$ y $\mathrm{Al}$ con $1.23 \%$ y $0.68 \%$ en peso respectivamente.
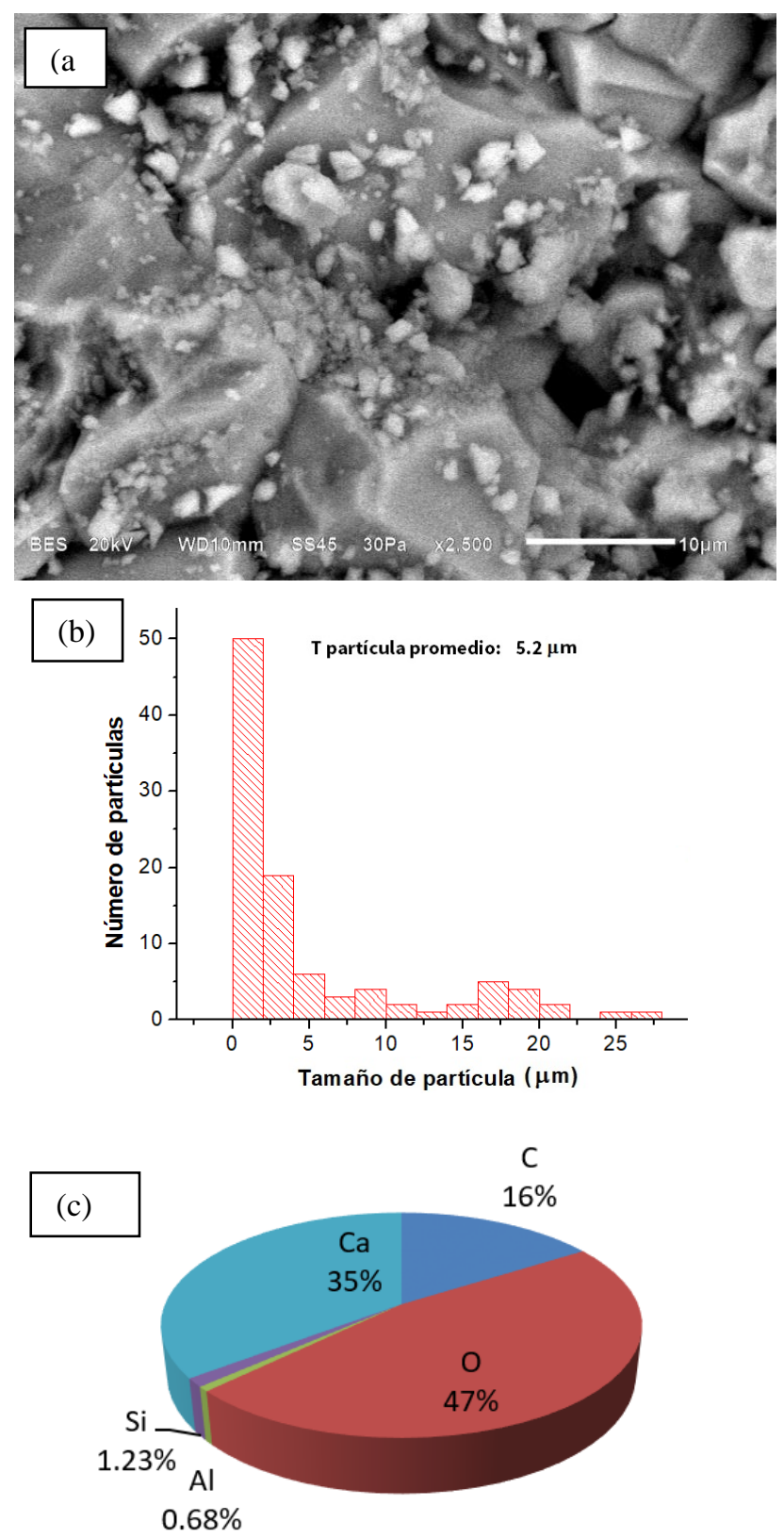

Figura 8. (a) Micrografía de la muestra M4, (b) Histograma del tamaño de grano correspondiente a la muestra M4, (c) Análisis químico elemental en \% en peso de la muestra M4.

En la Figura 9 se ilustran los patrones de difracción de las muestras M1 a M4, en todos los patrones los picos de difracción presentes corresponden a la fase cristalina de la calcita, de acuerdo a la tarjeta de indexación PDF 02-0629 de la base de datos PDF4+ del 2013 del Centro Internacional de Datos de Difracción (ICDD). 


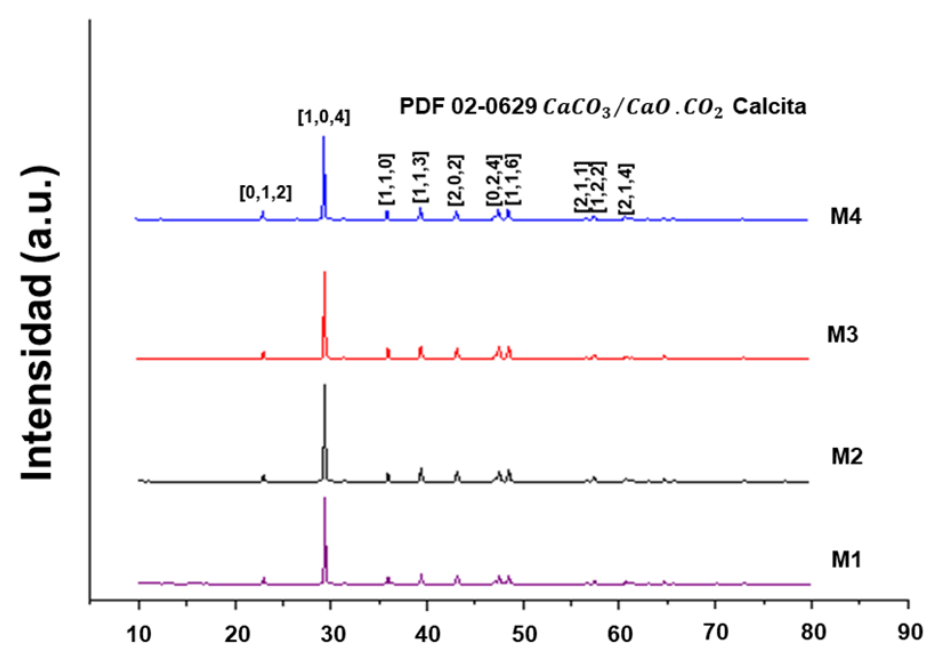

Figura 10. Patrones de difracción de rayos X, de las muestras M1, M2, M3 y M4, obtenidas de una cantera de Tepexi de Rodríguez, Puebla, las muestras presentan la fase calcita de acuerdo a la tarjeta de indexación PDF 02-0629

En la Figura 10 se presentan los espectros en el infrarrojo asociados a las muestras M1 a M4, en los cuales se muestran las bandas correspondientes a las vibraciones de estiramiento perteneciente a los grupos C-O localizadas en $1396 \mathrm{~cm}^{-1}$, de igual forma se visualizan las bandas situadas a $1030 \mathrm{~cm}^{-1}$ asociadas a la presencia de dolomita, las bandas correspondientes a $872 \mathrm{~cm}^{-1}$ son atribuidas a vibraciones fuera del plano de los grupos C-O. Finalmente las bandas localizadas en $712.20 \mathrm{~cm}^{-1}$, son asociadas a vibraciones en el plano de los grupos C-O. Estas bandas corresponden a la presencia de calcita en concordancia con los resultados de difracción de rayos $\mathrm{X}$.

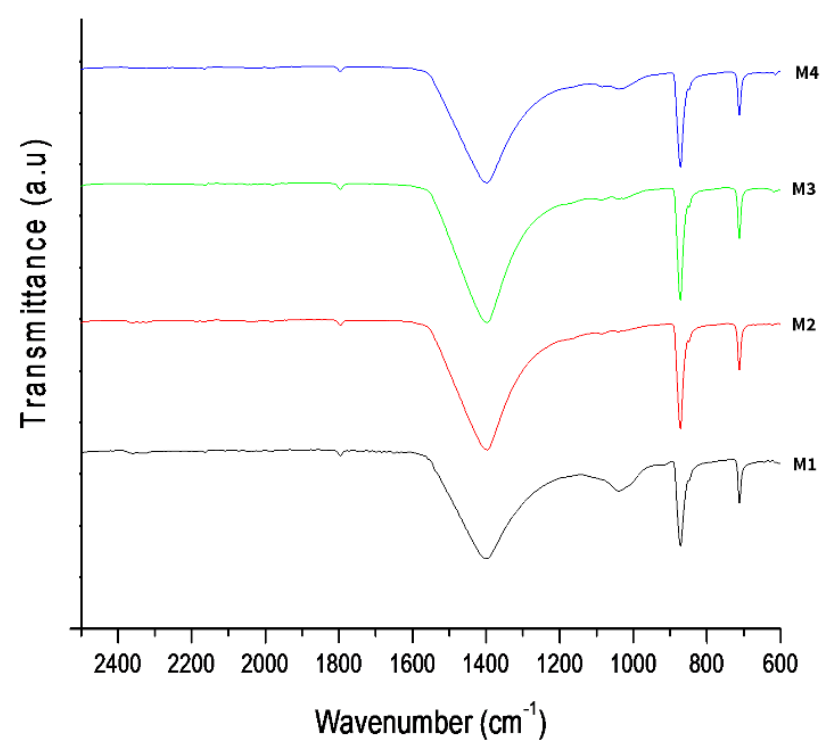

Figura 9. Espectros en el infrarrojo (FTIR) de las muestras M1, M2, M3 y M4, obtenidas de una cantera de Tepexi de Rodríguez, Puebla.

\section{Conclusiones}

Los resultados por microscopía electrónica de barrido nos permiten concluir que cada muestra que se estudió de los diferentes estratos tienen cambios significativos en el tamaño de grano, siendo la muestra M4 la que presenta un mayor tamaño de grano por arriba de los 25 micrones y por otra parte las muestras M1, M2 y M3 son las que presentan un tamaño inferior a las cinco micras, con este resultado, podemos anticipar que las propiedades de resistencia a los esfuerzos de compresión deben tener cambios significativos, así como la resistencia a la abrasión. Los resultados por difracción de rayos X y por espectroscopia en el infrarrojo (FTIR), permiten confirmar la presencia mayoritaria de la fase calcita, sin embargo, por la presencia de elementos químico minoritarios como $\mathrm{Si}, \mathrm{Al} \mathrm{y} \mathrm{Mg}$, detectados por espectroscopia de dispersión de rayos $\mathrm{X}$, es posible asegurar la presencia de otras fases cristalinas en concentraciones inferiores al $1 \%$, que no fueron detectadas a consecuencia de límite de detección de los equipos utilizados. Finalmente, estos resultados demuestran que un estudió de esta naturaleza o algunos estudios de resistencia a los esfuerzos son importantes considerar para las diferentes aplicaciones del mármol, particularmente en piezas que van a estar sometidas a la abrasión constante.

\section{Referencias}

Corinaldesi, Valeria, Giacomo Moriconi, and Tarun R. Naik. 2010 "Characterization of Marble Powder for Its Use in Mortar and Concrete." Construction and Building Materials 24(1):113-17.

Hernández, Juan, Eleazar Salinas, Alberto Blanco, Eduardo Cerecedo, and Ventura Rodríguez. 2014. Carbonato de Calcio En México. Omnia.

Miranda-Trejo, Mario, Ignacio Ocampo-Fletes, Juan F. Escobedo-Castillo, and Ma. Lourdes Hernàndez-Rodrìguez. 2015. "La Distribución Del Agua Potable En Tepexi de Rodríguez, Puebla." Agricultura, Sociedad y Desarrollo 12:261-77.

Morales Tassinai, Adolfo Manuel, Javier Guevara Rivera, Pedro Cruz Ortega, and Jorge Arturo Hernández Zárate. 2017a. "Estudio de Alteración Del Peso En El Mármol Tipo Café Tabaco Mediante Una Prueba de Hume." Ingeniantes 1(1):21-27.

Morales Tassinai, Adolfo Manuel, Javier Guevara Rivera, Pedro Cruz Ortega, and Jorge Arturo Hernández Zárate. 2017b. "Medición de La Alteración Del Peso En El Mármol Tipo Café Tabaco Mediante Una Prueba de Cristalización de Sales Por Cloruro de Sodio." Ingeniantes 1.

Morales Tassinai, Adolfo Manuel, María Esperanza Velasco Ordónez, and Jaime Ramírez Peña. 2019. "Modelo de Enfriamiento En El Mármol Tipo Café Tabaco Mediante Una Prueba de Enfriamiento de Newton." Iberoamericana de Ingeniería Mecánica 23:35-44.

Morales Tassinai, Adolfo Manuel, María Esperanza Velasco Ordónez, and Jaime Ramírez Peña. 2020. "Estudio Comparativo de Alteración Del Peso En El Mármol Tipo Café Tabaco Mediante Ensayos de Cristalización de Sales Por Sulfato de Sodio." Iberoamericana de Ingeniería Mecánica 24:69-80.

Rioja, L. A., Santo Domingo, and De Calzada. 2016. Análisis Químico , Diagnóstico y Propuestas de Conservación-Restauración Para La Muralla de Santo Domingo de La Calzada.

Semar. Gamas y Colores del Mármol (28 mayo, 2019). https://pymsemar.com/. Recuperado el 12 de octubre de 2020 de https://pymsemar.com/gamas-y-colores-del marmol/\#: :text=Los\%20colores\%20del $\% 20 \mathrm{~m} \% \mathrm{C} 3 \% \mathrm{~A} 1 \mathrm{rmol} \% 20 \mathrm{ma}$ $\mathrm{s}, \mathrm{m} \% \mathrm{C} 3 \% \mathrm{~A} 1 \mathrm{~s} \% 20 u$ tilizados\%20en\%20el\%20mundo.

Tlatempa, M. T., R. F. Estrada, E. Rubio, and V. Rodríguez Lugo. 2011.

"Microstructural Characterization of Marble from the Quarry Coffee Tobacco Proceeding from Tepexi de Rodriguez, Puebla." Acta Microscopica 20(1). 